\title{
Dengue Encephalitis---A Rare Entity
}

\author{
Sourya Acharya ${ }^{1}$, Samarth Shukla ${ }^{2}$, Rasika Thakre ${ }^{3}$, Nirmesh Kothari ${ }^{4}$, SN \\ Mahajan ${ }^{5}$ \\ ${ }^{1}$ Professor. Dept of Medicine, ${ }^{2}$ Professor. Dept of Pathology, ${ }^{3}$ Resident. Dept of Medicine,${ }^{4}$ Asst. Prof. Dept of \\ Medicine, ${ }^{5}$ Prof \& HOD. Dept of Medicine
}

Abstract: Encephalitis is an uncommon manifestation of dengue fever. We present a case of a 30 year old female who presented with severe encephalitis due to dengue infection.

Key words: encephalitis, dengue

\section{Introduction:}

Dengue fever has varying clinical presentations ranging from asymptomatic infection to life threatening hemorrhagic fever and dengue shock syndrome. Dengue is an increasingly prevalent arboviral infection common in tropical countries including South and Southeast Asia. Fever, arthralgia, headache, petechial spots, rash and hemorrhagic manifestations are common features. However, neurologic complications in general are unusual. ${ }^{1}$

\section{Case Report:}

A 30 year old , unmarried female presented to us with chief complaints of fever, headache with on and off episodes of vomiting since 8 days duration, one episode of generalized tonic clonic seizures and altered sensorium since one day. On examination she was febrile with axillary temperature of $102.8^{0} \mathrm{~F}$, pulse was 128/min, pallor, icterus were absent. There was no significant lymphadenoipathy, rash or edema. CVS , RS and per abdomen examination was normal. In CNS examination, she was conscious, irritable, not responding to verbal commands but was moving all four limbs to deep painful stimuli. Brain stem signs were normal. DTR was exaggerated in upper and lower limbs, hypertonia was present in all four limbs and plantars were bilateral extensor. Mild neck stiffness was present.

Investigations revealed $\mathrm{Hb}$ of $11 \mathrm{gm} \%$. The platelet count was $46,000 / \mathrm{mm} 3$, WBC was $6,200 / \mathrm{mm} 3$ with a normal differential count and normal WBC morphology. The serum sodium levelwas $128 \mathrm{mEq} / \mathrm{l}$, SGPT was $200 \mathrm{IU} / \mathrm{l}$, SGOT $250 \mathrm{IU} / \mathrm{l}$, serum bilirubin was $0.8 \mathrm{mg} / \mathrm{dl}$, serum albumin was $2.5 \mathrm{~g} / \mathrm{dl}$, DIC profile was mildly deranged and the blood urea and serum creatinine were normal. The cerebrospinal fluid (CSF) revealed a normal opening pressure, protein was $70 \mathrm{mg} / \mathrm{dl}$ and all 30 cells $/ \mathrm{mm} 3$ were lymphocytes. Gram staining, ZichlNeelsen staining and fungal staining were negative. CSF adenosine deaminase (ADA) was normal and ELISA for Japanese and herpes encephalitis were both negative. Paired sera for dengue were positive for IgM antibodies. The CSF was positive for dengue IgM antibody. Blood cultures, urine culture and CSF culture were negative. ELISA for leptospirosis was negative. A chest $\mathrm{x}$-ray and USG abdomen were normal. A Widal test(Span Diagnostic, India), rapid slide test for malaria (OptiMAL) and exam for typhoid were negative. Virus isolation and typing was not done due. MRI FLAIR image of brain showed bilateral symmetrical hyperintensity in gangliocapsular region (fig-1) and around the temporal horns, midbrain and hippocampus. (fig-2)

She was treated with intravenous fluid, intravenous mannitol, iv anti epileptics, Paracetamol, and iv antibiotics for prevention of bacterial infections. Empirical iv methyl prednisolone $1 \mathrm{gm}$ was given for 3 days in view of extensive demyelination. There was no improvement in her neurological status after 2 weeks of hospitalization and she was discharged on request. 


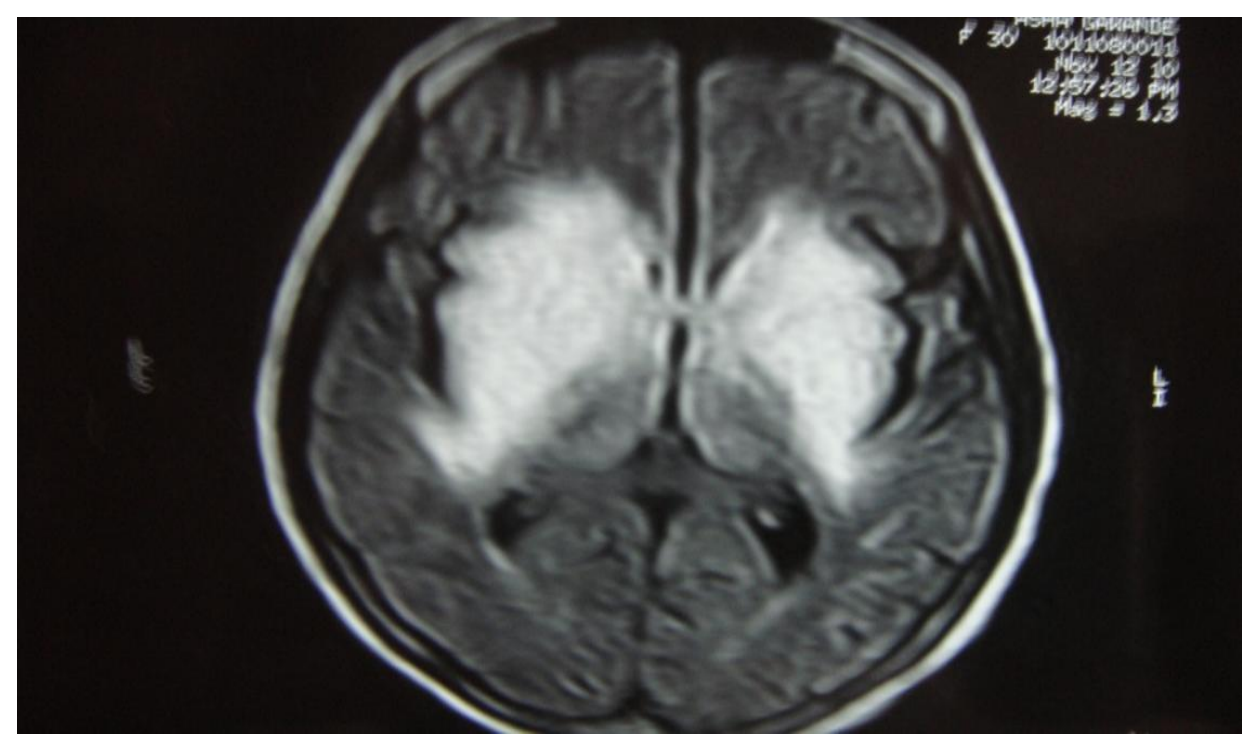

Fig 1: MR FLAIR image showing bilateral symmetrical hyperintensity in ganglio capsular region.

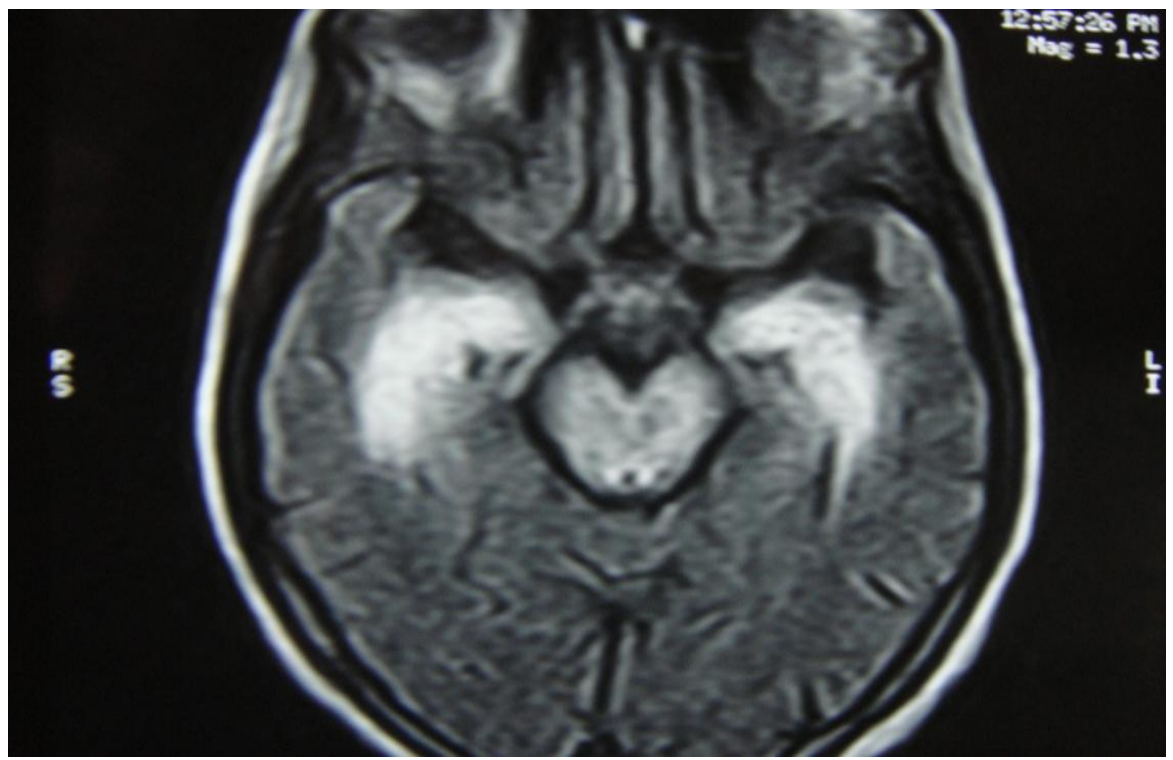

Fig 2: MR FLAIR image showing bilateral symmetrical periventricular hyperintensity around the temporal horns, mid-brain showing diffuse hyperintensity.

\section{Discussion:}

Most dengue cases are usually reported during epidemics in India and other parts of the world, though sporadic cases have been reported. The neuroinvasive properties of dengue virus are not well known but there are some reports of nervous system involvement in various parts of the world. ${ }^{2,3}$ Encephalitis in dengue fever is a rare entity. ${ }^{4,5}$ The various nervous system manifestations of dengue infection can be symptoms and signs predominantly involving the central nervous system like altered level of consciousness, seizures, pyramidal tract signs, meningeal signs, headache and encephalitis. The conditions associated with peripheral nervous system affection are myelitis and Gullain Barre syndrome. ${ }^{6,7}$ The exact pathophysiology of this neurovirulent property of the virus is not clear. Since in dengue fever the virus mainly replicates in cells of the macrophage line, infiltration of virus infected macrophages into the brain is one pathway of entry into the brain in dengue encephalitis. ${ }^{5}$ Dengue virus type 2 has been demonstrated in the CSF of a dengue encephalitis patient. ${ }^{8}$ Dengue virus serotype 4 has been detected by immunohistochemistry and by RT-PCR in inferior olivary nucleus of medulla and granular layers of cerebellum, furthermore, immunoreactivity has been observed in endothelial cells, astrocytes, neurons and microglia. ${ }^{1}$ Extended immunohistochemical studies have shown the virus positive cells located mostly with Virchow Robin space of medium size and small veins, infiltrating the white and gray matter are often close to neurons displaying cytopathic features. However, it is not clear whether virus infected macrophages or virus free particles cause the lesions in nervous system by immune, metabolic and/or direct cytopathic effect. 
MRI shows various focal and diffuse lesions in different studies involving mid brain, thalamus, hippocampi, temporal lobes, pons, and spinal cord. In our case demyelination was also seen in bilateral gangliocapsular regions which has not been reported so far as a site of involvement. Clearly, much of the data is disparate and a conclusive characterization of the MRI features of dengue encephalitis is not yet possible, although the focal nature of imaging abnormalities adds weight to the theory of viral neurotropism.

General management of viral encephalitis includes monitoring and maintenance of the airway and of adequate oxygenation, hydration, and nutrition. Seizures may be controlled by standard anti-epileptic drugs, and raised intracranial pressure by head-up nursing, mannitol, and steroids. ${ }^{13}$ If bacterial infection remains a possibility then empirical antibiotics appropriate to local organisms should be given. In endemic areas other CNS infections, including cerebral malaria, toxoplasmosis, neurocysticercosis, human immunodeficiency virus (HIV), and tuberculosis should also be excluded, along with local viruses, for example, Japanese encephalitis in Asia and West Nile virus in Africa. A high index of suspicion is important to arrive at the correct diagnosis.

\section{References}

[1] Brain of a fatal case of haemorrhagic dengue fever. J Neurovirology 1998;4:465-8

[2] Mehendale SM, Rodringues FM, Pinto BD. A sporadic case of dengue encephalopathy. J Assoc Physicians India 1989; $37: 346$

[3] Witayathawornwong P. Fatal dengue encephalitis. Southeast Asian J Trop Med Public Health 2005;

[4] Chotmongkol V, Sawanyawisuth K. Case report: Dengue hemorrhagic fever with encephalopathy in an adult. Southeast Asian J Trop Med Public Health 2004; 35: 160-1.

[5] Koley TK, Jain S, Sharma H, et al. Dengue encephalitis. J Assoc Physicians India 2003; 51: 422-3.

[6] Soares CN, Faria LC, Peralta JM, Defreitas MR, Puccioni-Sohler M. Dengue infection: neurological manifestation and cerebrospinal fluid (CSF) analysis. J Neurol Sci 2006; 249: 19-24.

[7] Thisyakorn U, Thisyakorn C, Limpitkul W, et al. Dengue infection with cerebral nervous system manifestations. Southeast Asian J Trop Med Public Health 1999; 30: 504-6.

[8] Hommel D, Talarmin A, Deubel V, et al. Dengue encephalitis in French Guiana. Res Virol 1998;149:235-8.

[9] Cam BV, Fonsmark L, Hue NB, Phuong NT, Poulsen A, Heegaard ED. Prospective case-control study of encephalopathy in children with dengue hemorrhagic fever. Am J Trop Med Hyg 2001;65:848-51.

[10] Misra UK, Kalita J, Syam UK, Dhole TN. Neurological manifestations of dengue virus infection. J Neurol Sci 2006;244:117-22.

[11] Kamble R, Peruvamba JN, Kovoor J, Ravishankar S, Kolar BS. Bilateral thalamic involvement in dengue infection. Neurol India 2007;55:418-9.

[12] Yeo PS, Pinheiro L, Tong P, Lim PL, Sitoh YY. Hippocampal involvement in dengue fever. Singapore Med J 2005;46:647-50.

[13] Solomon T, Hart IJ, Beeching NJ. Viral encephalitis: A clinician's guide. Pract Neurol 2007;7:285-302. 